\title{
Incomplete sulphomucin-secreting intestinal metaplasia for gastric cancer. Preliminary data from a prospective study from three centres
}

\author{
M I FILIPE, F POTET, W V BOGOMOLETZ, P A DAWSON, B FABIANI, \\ P CHAUVEINC, A FENZY, B GAZZARD, D GOLDFAIN, AND R ZEEGEN \\ From the Department of Histopathology, Guy's Hospital Medical School, London, Service Central \\ d'Anatomi Pathologique, Hôpital Beaujon Clichy-INSERM U239, Faculté X. Bichat, Paris and Laboratoire \\ d'Anatomie Pathologique, Institut Jean Godinot, Reims Groupe Medical St Remi, Reims; Dreux; and \\ Westminster Hospital, London
}

SUMMARY A joint prospective long term study of gastric biopsies has been undertaken to survey intestinal metaplasia Types I, II, and III in terms of their incidence, distribution and value in the selection of high risk cancer patients. This study is based on protocols agreed between three centres for endoscopy, histological interpretation, and mucin histochemistry. The results on the first 1350 gastric biopsies examined during 1981-1982 are presented. Intestinal metaplasia was found in 267 biopsies (20\%), being common in association with carcinoma $(71 \%)$, less so in benign conditions such as gastric ulcer $(39 \%)$, and chronic gastritis $(24 \%)$, but rare in 'normal' (3\%). Type I intestinal metaplasia was predominant $(73 \%)$ in all the biopsies with intestinal metaplasia and was most common in benign conditions, $70 \%$ in gastric ulcer and $76 \%$ in chronic gastritis. Type III intestinal metaplasia (incomplete sulphomucin-secreting intestinal metaplasia) was recorded in only $9.8 \%$ of all the biopsies with intestinal metaplasia and had a higher incidence in carcinoma $(35 \%)$, than in benign conditions $(7 \%)(p<0.0001)$. These results suggest that intestinal metaplasia types may have different malignant potential and their identification may be useful in screening patients for early detection of cancer.

Intestinal metaplasia is generally considered a premalignant lesion. ${ }^{1}$ Its common occurrence and presence in benign and malignant conditions, however, limits its value as indicator of gastric cancer risk.

Recent studies have identified types of intestinal metaplasia which may have different malignant potential. An association between a variant of the intestinal metaplasia with incomplete cell differentiation and sulphomucin secretion and the intestinal type gastric carcinoma has been suggested..$^{2-7}$ These observations are based on retrospective examination of gastrectomy and biopsy material.

A prospective study on gastric biopsies with follow up of patients was needed to assess the incidence and distribution of intestinal metaplasia

Address for correspondence: M I Filipe, Senior Lecturer/Hon. Consultant, Department of Histopathology, Guy's Hospital Medical School, London Bridge, London SE1 9RT

Received for publication 1 March 1985 types and to establish their value in the selection of patients at high risk for developing cancer.

We have undertaken such a project involving three centres: London (Westminster and Guy's Hospitals), Paris (Hôpital Beaujon) and Reims (Institut Jean Godinot). It is intended to follow up patients for at least five years.

We report here the preliminary results on 1350 gastric biopsies examined during the first two years: 1981 and 1982.

\section{Methods}

PATIENTS

The centres involved are not in areas of high incidence of gastric cancer.

Biopsies were obtained from patients with gastric symptoms attending the endoscopy clinics. There was no selection of patients with any particular type of gastric condition. Healthy controls were not 
included. The age range was 19-91 years (mean 56.8 years) with predominance of men (M/F 1:6).

\section{ENDOSCOPY PROTOCOL}

A protocol was discussed with the endoscopists who agreed to participate in this study in the three centres. Particulars of the patient, age, and sex were noted. In summary, the protocol included: (a) endoscopic findings: gastritis, ulcer etc; (b) site of biopsy; (c) number of specimens.

Multiple gastric biopsies, whenever possible, were taken from the lesion, and from areas adjacent and remote from it. In the absence of gross lesion, random biopsies were obtained from the various gastric regions, including fundus and antrum. On average 3.7 biopsies were taken on each occasion from each patient (counted as one biopsy, in this study), each specimen measuring $3 \mathrm{~mm} \times 2 \mathrm{~mm}$ on average.

\section{HISTOLOGY AND MUCIN HISTOCHEMISTRY:}

\section{PROTOCOL AND METHODS}

A common protocol for the histological interpretation of the biopsies was used in the three centres.

All material was fixed in $10 \%$ formol saline and routinely embedded in paraffin wax. Serial sections were cut, stained with haematoxylin-eosin and the following techniques for mucins: Alcian blue $\mathrm{pH} 2 \cdot 5-$ periodic acid-Schiff (AB/PAS) and high irondiamine plus Alcian blue $\mathrm{pH} 2.5$ (HID/AB), to identify neutral, sialo- and sulphomucins. ${ }^{8}$

Biopsies which were too superficial or small and/or contained no gastric mucosa - for example, slough, granulation tissue or tumour only, were excluded. Biopsies from the gastro-oesophageal junction and from the gastric stump in postgastrectomy patients were also excluded.

Morphological and histochemical features were recorded according to the protocol as follows (summary): (a) number of specimens (with and without intestinal metaplasia), orientation, presence or absence of muscularis mucosae: (b) mucosal type; (c) histology graded as 'apparently normal', gastritis (with or without ulcer), dysplasia, neoplasia, other; (d) Intestinal metaplasia, (histochemical characterisation). Gastritis was classified according to the criteria proposed by Morson and Dawson ${ }^{9}$ and intestinal metaplasia was classified according to Jass and Filipe. ${ }^{46}$

Type I (complete): mature absorptive and goblet cells, the latter secreting sialomucins. (Fig. 1).

Type II (incomplete): few or absent absorptive cells; presence of columnar 'intermediate' cells in various stages of differentiation, secreting neutral and acid sialomucins; goblet cells secreting sialomucins and/or occasionally sulphomucins (Fig. 2).

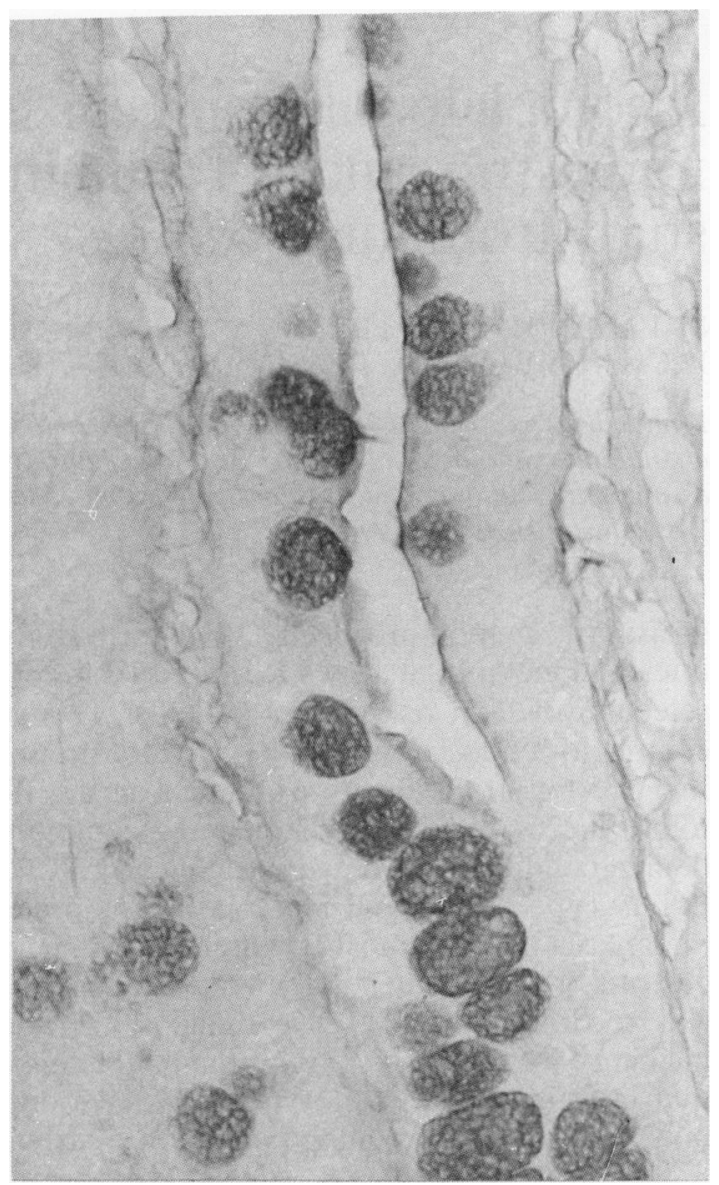

Fig. 1 Gastric biopsy. Intestinal metaplasia Type I: Goblet cells secreting sialomucins and mature non-secretory absorptive cells in between. ('Blue' HID-AB method).

Type III (incomplete): cell dedifferentiation is more marked than in type II; 'intermediate' cells secrete predominantly sulphomucins; goblet cells secrete sialo- and/or sulphomucins. A variable degree of disorganised glandular architecture is often present (Fig. 3). (In the original classification $^{46}$ types II and III were termed IIA and IIB respectively.)

Forms of transition in terms of morphology and mucin profile between these three well recognisable variants were also noted and described in more detail in the Results below.

To standardise the histological and the intestinal metaplasia types criteria, the following steps were taken: (a) assessment of the slides by the three senior pathologists (MIF, FP, and WVB) using a double headed microscope at the start of the study 


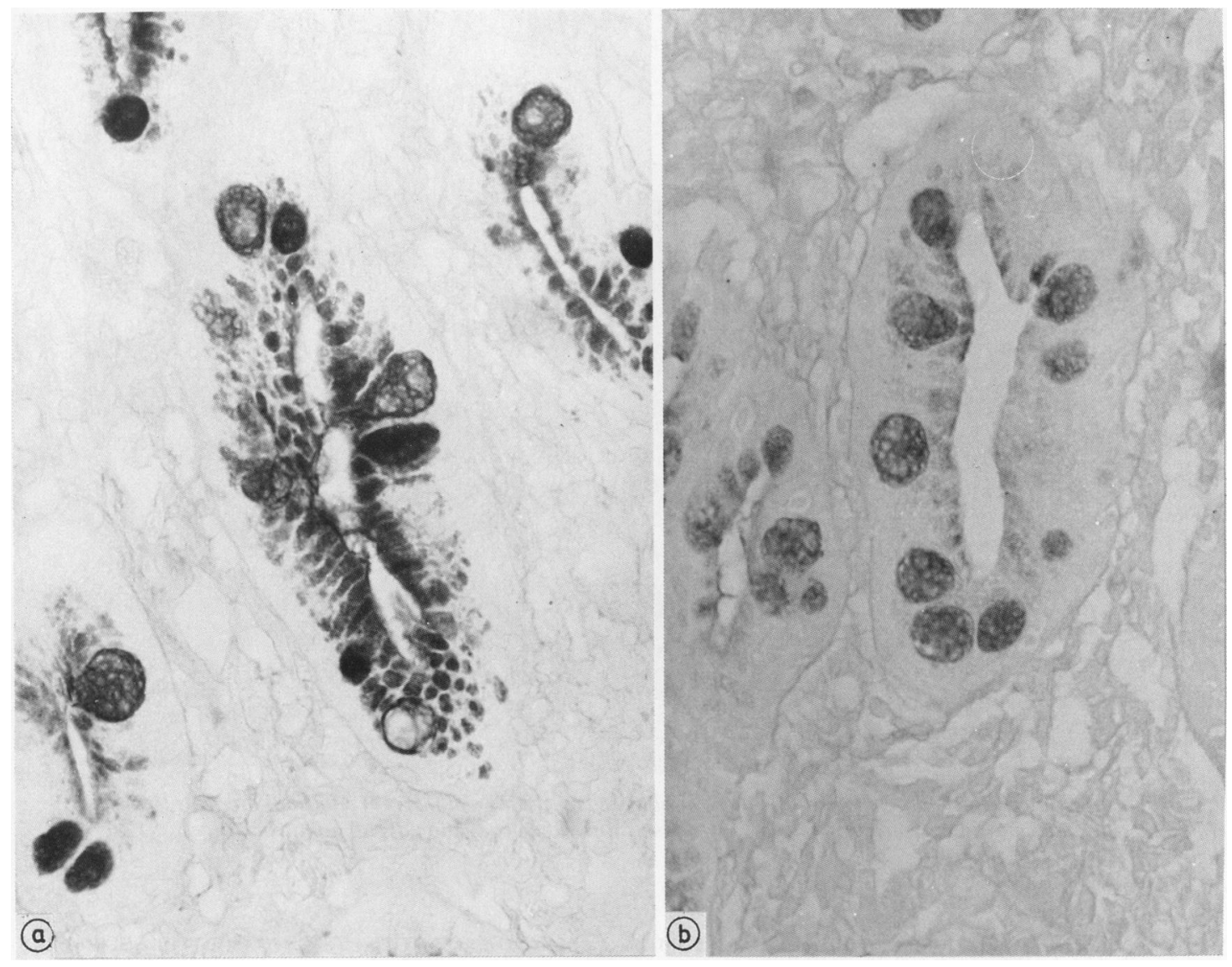

Fig. 2 Gastric biopsy. Intestinal metaplasia Type II: section a on Alcian blue-PAS staining, goblet cells secreting acid mucins and columnar 'intermediate' cells in between, contain a mixture of neutral and acid mucins. On section b stained with HID-AB, mucin in 'intermediate' cells is predominantly neutral mucin (unstained) and scanty sialomucin (pale grey).

and several times during its course; (b) slides were also exchanged for double blind assessment; (c) to standardise the histochemical techniques, technicians were trained in one of the centres (MIF) and the same source of reagents was used in all three centres; (d) quality staining control checks were carried out at various intervals.

For statistical analysis $\chi^{2}$ contingency tables were used. Fisher's exact test was used for small numbers.

\section{Results}

One thousand three hundred and fifty gastric biopsies were examined.

\section{(1) DISTRIBUTION OF GASTRIC CONDITIONS}

(TABLE 1)

Over one third of the biopsies $(36 \%)$ were reported as 'apparently normal', having no histological abnormalities and about half $(48 \%)$ showed true chronic gastritis either superficial, or atrophic, not associated with gastric ulcer. Gastric ulcer and gastric carcinoma accounted respectively for $15 \%$ and $2 \%$ of the patients. The latter values, however, do not represent the true incidence of these two lesions in the material as, according to our criteria, only those biopsies showing gastric epithelium were included in the analysis of results.

The following differences were noted between the three centres. $\left(\chi_{6}^{2}=221 \cdot 4 ; \mathrm{p}<10^{-6}\right)$.

The proportion of 'apparently normal' biopsies was much higher in Paris and Reims (35\% and 56\% respectively) than in London (15\%) and Paris also showed a higher incidence of chronic gastritis $(56 \%)$. In contrast gastric ulcer was a more common finding in the London material $(42 \%)$ compared 


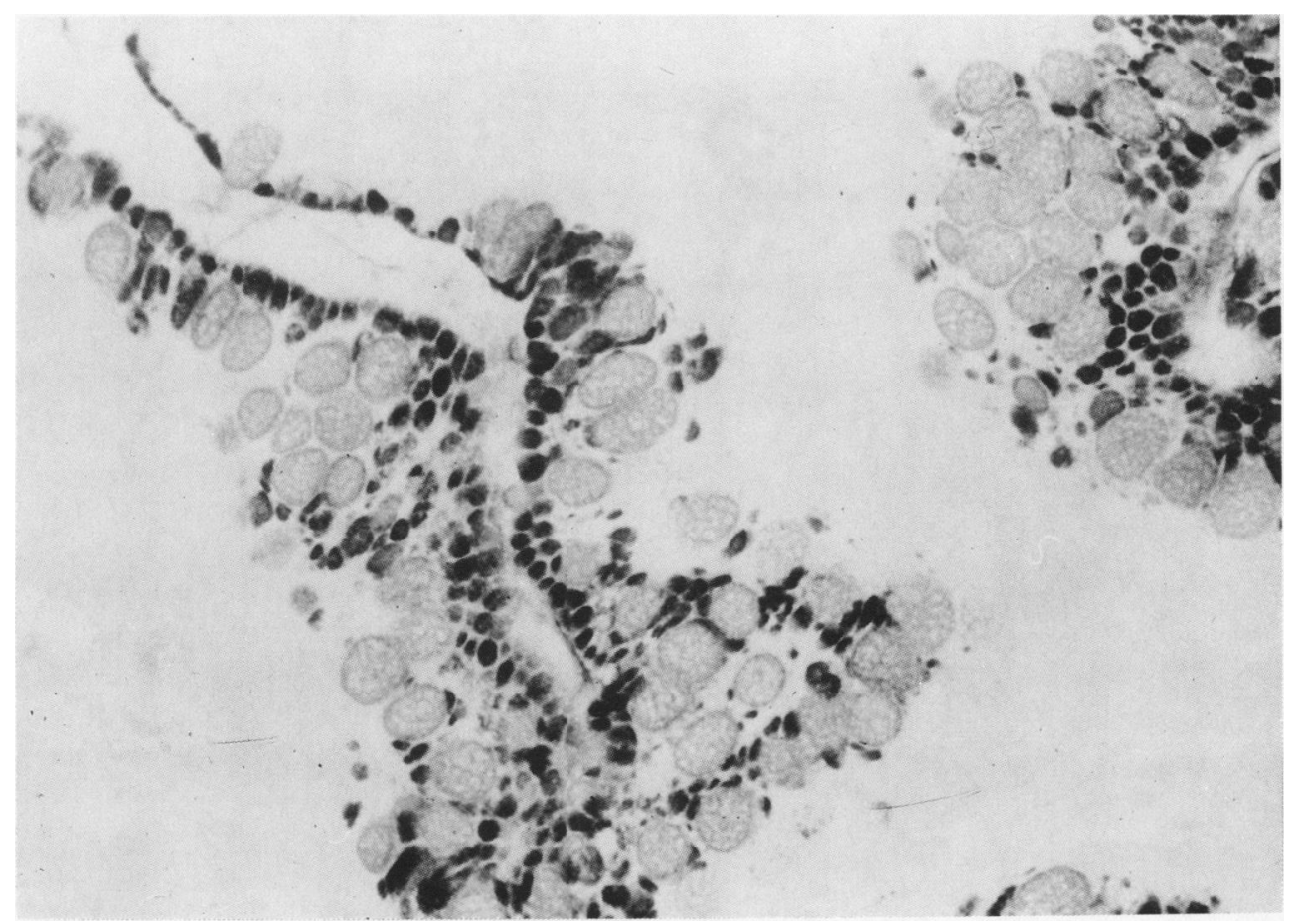

Fig. 3 Gastric biopsy. Intestinal metaplasia Type III: goblet cells secreting sialo- (grey) and sulphomucins (black); columnar 'intermediate' mucus-secreting cells in between show marked production of sulphomucins (black). (HID-AB method).

with the other two centres (8\% and $13 \%)$. Regarding gastric carcinoma no significant difference was observed between London and Paris, whereas one case only was included in the series from Reims.

Table 1 Histological data in 1350 gastric biopsies (1981 and 1982) from three centres: London, Paris and Reims

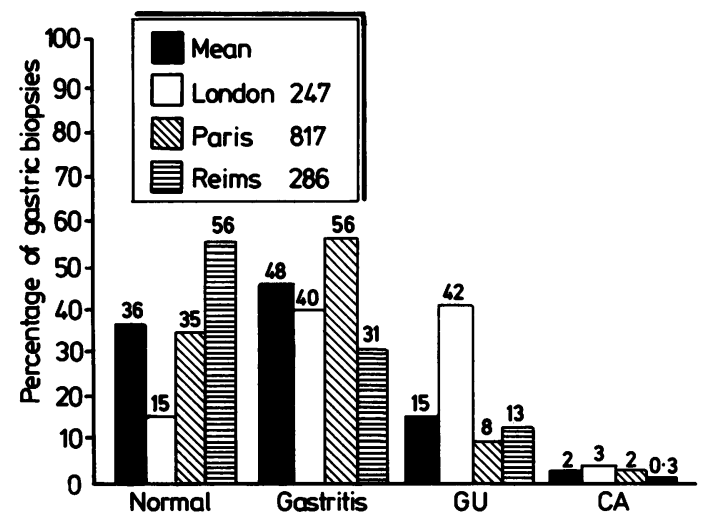

$\mathrm{GU}=$ gastric ulcer, $\mathrm{CA}=$ carcinoma.
(2) INCIDENCE OF INTESTINAL METAPLASIA TYPES (TABLES 2, 3 AND 4)

Intestinal metaplasia was found in $20 \%$ of the biopsies in benign conditions and carcinoma. It was

Table 2 Incidence of intestinal metaplasia in 1350 gastric biopsies

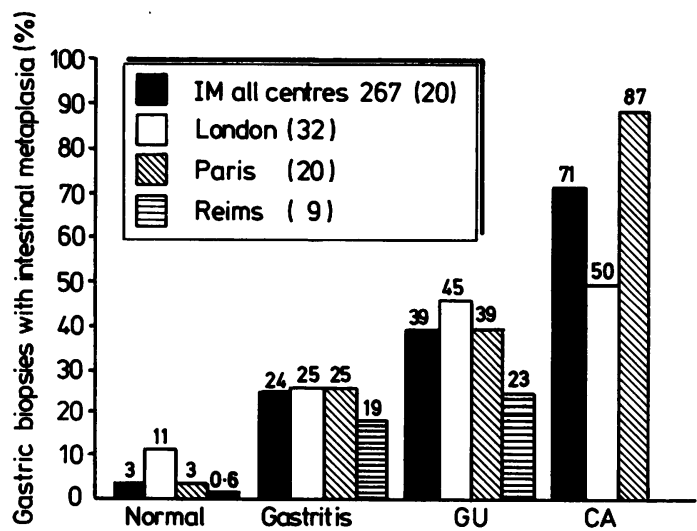

IM=intestinal metaplasia 
Table 3 Intestinal metaplasia types - incidence in 267 biopsies with intestinal metaplasia

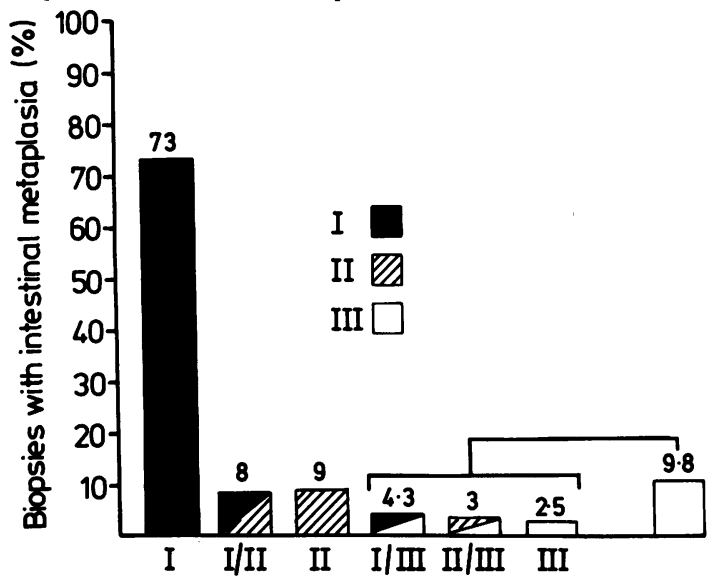

common in association with carcinoma $(71 \%)$ less so in gastric ulcer (39\%), chronic gastritis $(24 \%)$ and rare in 'apparently normal' (3\%). (Table 2).

Intestinal metaplasia of all types was more often found in biopsies from the antrum $(93 \%)$ than in those from the fundus. In the fundus, the incidence of the various intestinal metaplasia types was similar

Table 4 Intestinal metaplasia types: comparison between centres

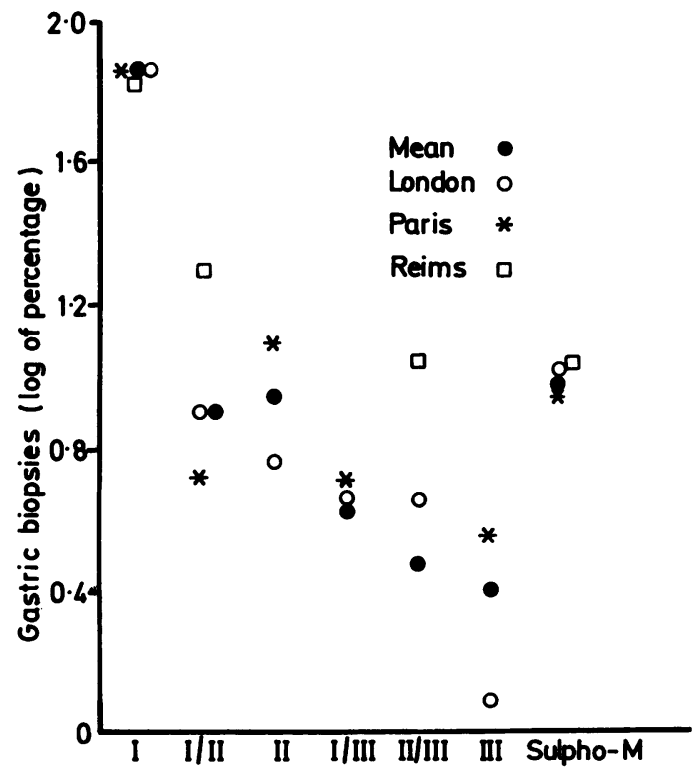

(7\%, $11 \%$ and $4 \%$ for Types I, II and III respectively).

As shown in Table 3, Type I intestinal metaplasia was predominant $(73 \%)$ whereas Type III variants secreting sulphomucins were seen in only $9.8 \%$ of the biopsies with intestinal metaplasia. In Type III variants we included cases of typical Type III having marked cellular dedifferentiation and high sulphomucin content in the 'intermediate' cells, as well as cases of less well defined incomplete intestinal metaplasia where small quantities of sulphated material are demonstrated in fine apical granules in the 'intermediate' cells, and often involving single or few glands in areas of predominantly Type I (I/III) or II (II/III).

Type II (well defined Type II and variants I/II) accounted for $17 \%$ of intestinal metaplasia seen. (Table 3).

In spite of the higher proportion of biopsies with intestinal metaplasia in the London series $(32 \%)$ than in the Paris group (20\%), there were no significant differences between the two centres regarding the incidence of the intestinal metaplasia in the various gastric conditions with exception of 'apparently normal' biopsies $(11 \%$ and $3 \%$ in London and Paris respectively; $\left(\chi_{2}^{2}=9.457\right.$; $\mathrm{p}<0.0088)$, but not in carcinoma $\left(\chi_{2}^{2}=6 \cdot 104\right.$; $p<0.053)$ ). The variations in the incidence of intestinal metaplasia types (I; II and variants; III and variants) between the three centres were not significant (Table $4 ; \chi_{4}^{2}=1 \cdot 214$ ).

(3) DISTRIBUTION OF INTESTINAL METAPLASIA TYPES IN VARIOUS GASTRIC CONDITIONS (TABLES 5 AND 6 )

Type I intestinal metaplasia was most common in benign conditions $(92 \%, 76 \%$, and $70 \%$ respectively in 'apparently normal', chronic gastritis and gastric ulcer). Type III showed a higher incidence in carcinoma $(35 \%)$ than in gastric ulcer and chronic gastritis (11\% and $7 \%$ respectively) and none was found in the 'apparently normal' (Table 5). Consideration of Types I and II (non-sulphated) as a

Table 5 Distribution of intestinal metaplasia types I and III in gastric conditions

\begin{tabular}{lcccc} 
& $\begin{array}{c}\text { Biopsies } \\
\text { (No) }\end{array}$ & Total IM (\%) & $\begin{array}{c}\text { IM percentage types out } \\
\text { of total IM }\end{array}$ \\
\cline { 2 - 5 } & & & IM I & IM III \\
Normal & 480 & 3 & 92 & 0 \\
Gastritis & 642 & 24 & 76 & 7 \\
GU & 204 & 39 & 70 & 11 \\
CA & 26 & 71 & 29 & 35 \\
\hline Total & 1350 & 20 & 73 & 9.8
\end{tabular}


Table 6 Incidence of type III intestinal metaplasia in benign conditions and carcinoma

\begin{tabular}{lcccc}
\hline & Type I and II & Type III & Total \\
\cline { 2 - 5 } & (Non-sulphated) & (Sulphated) & \\
\cline { 2 - 5 } Benign lesions & 232 & $(93 \%)$ & $18(7 \%)$ & 250 \\
Carcinomas & 11 & $(65 \%)$ & $6(35 \%)$ & 17 \\
\hline Total & 243 & 24 & 267
\end{tabular}

whole, showed them to be common in benign conditions $(93 \%)$ and in carcinoma $(65 \%)$. In contrast, only $7 \%$ of benign conditions with intestinal metaplasia were of type III (sulphated), compared with $35 \%$ in carcinoma: this difference is highly significant $(\mathrm{p}<0 \cdot 0001)$. (Table 6).
(4) INTESTINAL METAPLASIA AND AGE (TABLE 7) The age distribution shows a higher incidence of chronic gastritis above 40 years of age; no apparent difference in peptic ulcer in patients over 30 and carcinoma, as expected, was seen in those over 50 years and gradually increased with age.

Intestinal metaplasia was rarely seen in biopsies from patients under 30 years, and then gradually increased with age.

\section{Discussion}

We confirm that intestinal metaplasia, though common, has a higher incidence in gastric carcinoma $(71 \%)$ than in benign conditions. The lesion is too prevalent and not highly sensitive, however, being absent in a proportion of carcinomas, and therefore can not be used for screening purposes.

The higher incidence of intestinal metaplasia variants with incomplete cell differentiation and

Table 7 Age distribution of gastric conditions and intestinal metaplasia

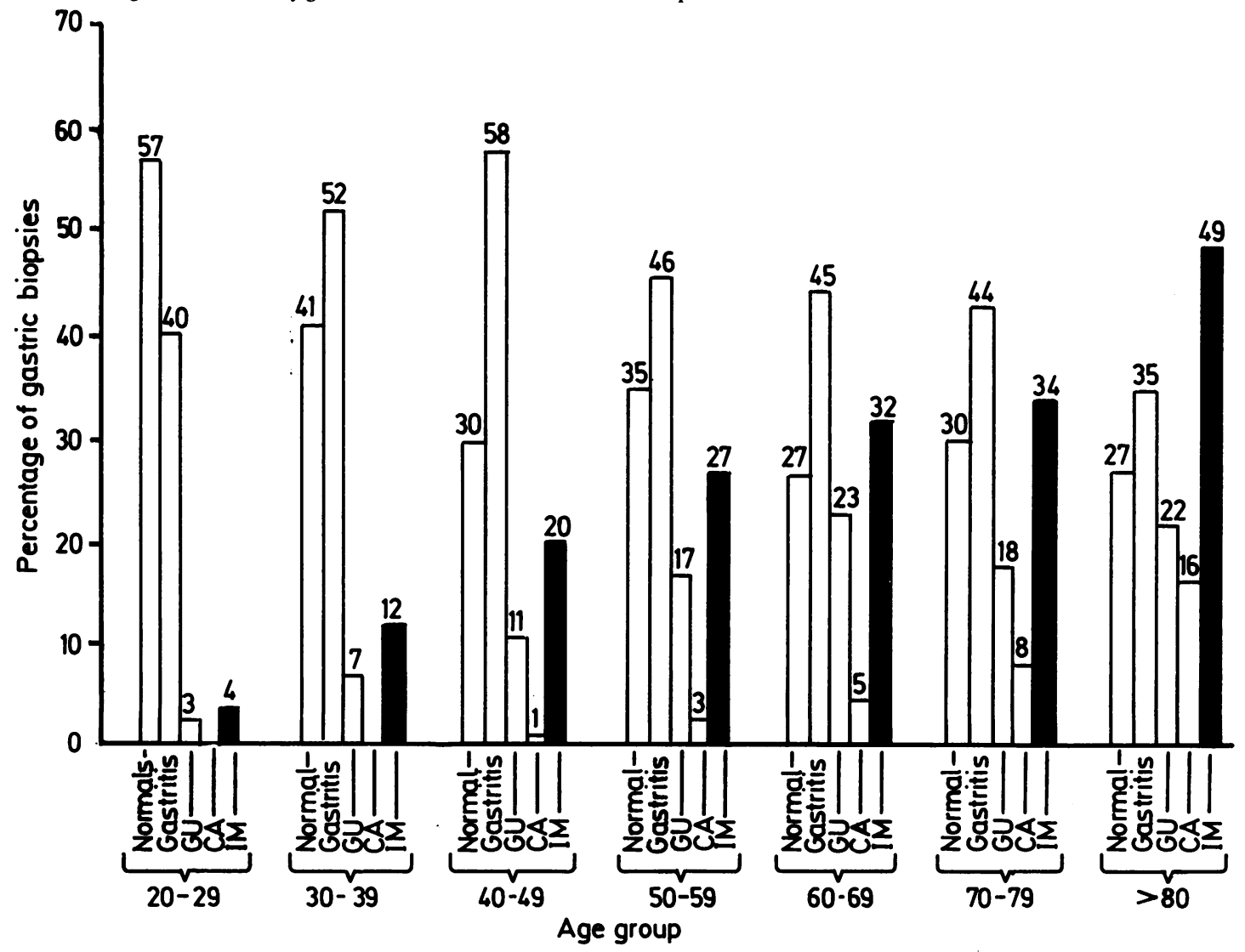


secreting sulphomucins in carcinomas (35\%) compared with only $7 \%$ in benign conditions was significant $(p<0.0001)$.

Several other authors have indicated that incomplete intestinal metaplasia with sulphomucins, which has been termed 'colonic' or 'enterocolic', could have a special role in the histogenesis of gastric cancer. $^{2} 55_{1011}$ The present results support this view.

Our figures for incomplete Type III intestinal metaplasia are very similar to those of Sipponen ${ }^{12}$ based on endoscopic biopsies of a large Finnish population: $31 \%$ for cancer (this study $35 \%$ ) and $6 \%$ for benign conditions (this study $7 \%$ ). It is interesting to note that similar studies from areas of high incidence of gastric cancer show a higher proportion of Type III intestinal metaplasia in benign conditions (39\%) and carcinoma $(50 \%)$ and in 11 of 12 cases of dysplasia. ${ }^{13} \mathrm{~A}$ good correlation is also found with gastrectomy material, where Type III intestinal metaplasia was seen in 49 to $57 \%$ of 'intestinal' and 'diffuse' carcinomas (Lauren's classification), and in only 5-8\% of benign conditions. ${ }^{4-16}$ As expected, the data for carcinoma are higher in the studies carried out on resected specimens than in endoscopic biopsies.

These various studies strongly suggest an association between Type III incomplete intestinal metaplasia and gastric carcinoma. In the present material a specificity of $93 \%$ is acceptable for screening purposes. A sensitivity of $35 \%$, however, is low.

Two factors may explain this low sensitivity. In general, intestinal metaplasia is more common and extensive in 'intestinal' than in 'diffuse' carcinomas. ${ }^{17} 18$ Moreover our observations on gastrectomy specimens seem to indicate that Type III incomplete intestinal metaplasia is associated with 'intestinal' carcinomas, both sharing a similar mucin profile. ${ }^{467}$ The other factor is sampling error and the topographical distribution of intestinal metaplasia Types. The difficulty of sampling focal lesions such as intestinal metaplasia and dysplasia is well recognised and accounts for a lower yield of intestinal metaplasia in biopsies. Multiple biopsies increase the accuracy, but equally important is a better knowledge of the likely regional distribution of the lesions. Indeed, the pattern of intestinal metaplasia distribution seems to depend on the cause of the underlying chronic gastritis ${ }^{11}{ }^{19}$ and also differs in gastric ulcer and carcinoma. ${ }^{4}$ Enzyme mapping techniques have shown that complete and incomplete Types of intestinal metaplasia have a preference for certain gastric zones and that incomplete intestinal metaplasia has a tendency to occupy particular zones within the stomach, irrespective of the presence, or absence of a carcinoma. ${ }^{1020}$ This information is important in endoscopy and in the interpretation of the significance of intestinal metaplasia Types in gastric biopsies.

This study confirms the higher incidence of intestinal metaplasia similarly to chronic gastritis, in the antrum, but failed to show any differential topographic distribution of certain intestinal metaplasia Types. The small number of carcinomas examined precludes any valid conclusion concerning patterns of intestinal metaplasia distribution and tumour site. ${ }^{11}$

Seven per cent of intestinal metaplasia in benign conditions was of Type III in this study. Whether or not these patients represent a group at higher risk of cancer can only be decided by a follow up study. There is strong evidence that chronic atrophic gastritis is a precancerous condition. ${ }^{1}{ }^{1921}$ It is intimately associated with intestinal metaplasia and a gradual change from chronic atrophic gastritis to intestinal metaplasia and finally to carcinoma has been reported. ${ }^{18}$ These changes are probably of environmental origin and it may take as long as 10-15 years for neoplasia to develop. ${ }^{22}$ We have not attempted, in this initial prospective study, to discriminate between types of chronic gastritis, nor did we separate 'intestinal' from 'diffuse' carcinomas as the numbers were too small.

Further support of the role of Type III intestinal metaplasia in the histogenesis of intestinal gastric cancer is its presence in adenomas but not in reactive polyps, ${ }^{23}$ and its high incidence in relatives of patients with gastric carcinoma and pernicious anaemia. ${ }^{5}$ Recent morphological observations have suggested that dysplastic changes appear in glands which already show intestinal metaplasia and that perhaps we should consider Type III intestinal metaplasia as a form of dysplasia. ${ }^{82425}$

The incidence of intestinal metaplasia increased with age in the group of patients reported here, an observation also reported by others. ${ }^{56}$ As intestinal metaplasia and carcinoma become more common with age, however, this finding is not a valid argument for, or against a causal relationship between the two lesions.

The disparity in the prevalence of certain gastric conditions between the centres, may possibly be explained by a different approach to the selection of patients and perhaps by different ethnic populations, environmental factors and the like, which we have not investigated.

In conclusion, an adequate long term follow up is necessary to assess the definite value of Type III intestinal metaplasia in the screening for gastric cancer and clarify the natural history of intestinal metaplasia Types. This is being done in the three centres. 
We would like to thank Miss P Brock, M C Durand, $M$ Perennec, and R Paris for their technical assistance; Dr F House, Guy's Hospital Medical School, London, for the statistical analysis and the Welton Trust, London, for financial support to P A Dawson. We are grateful to Dr H Thompson, General Hospital, Birmingham, for reading the manuscript and for his valuable comments.

\section{References}

1 Morson BC, Sobin LH, Grundmann E, Johansen A, Nagayo T, Serck-Hanssen A. Precancerous conditions and epithelial dysplasia in the stomach. J Clin Pathol 1980; 33: 711-21.

2 Teglbjaerg PS, Nielsen HO. 'Small intestinal type' and 'colonic type' intestinal metaplasia of the human stomach. Acta Pathol Microbiol Scand 1978; 86: 351-5.

3 Heilmann KL, Höpker WW. Loss of differentiation in intestinal metaplasia in cancerous stomachs. A comparative morphological study. Path Res Pract 1979; 164: 249-58.

4 Jass J, Filipe MI. A variant of intestinal metaplasia associated with gastric carcinoma: a histochemical study. Histopathology 1979; 3: 191-9.

5 Sipponen $\mathrm{P}$, Seppälä K, Varis K, et al. Intestinal metaplasia with colonic-type sulphomucins in the gastric mucosa; its association with gastric carcinoma. [Abstract]. Acta Pathol Microbiol Scand 1980; 88: 217-224.

6 Jass J, Filipe MI. The mucin profile of normal gastric epithelium, intestinal metaplasia and gastric carcinoma. Histochem J 1981; 13: 831-939.

7 Segura DI, Montero C. Histochemical characterization of different types of intestinal metaplasia in gastric mucosa. Cancer 1983; 52: 408-503.

8 Filipe MI, Lake BD. Histochemistry in pathology. London: Churchill Livingstone, 1983.

9 Morson BC, Dawson IMP. Gastrointestinal pathology. 2nd ed. London: Blackwell, 1979.

10 Matsukura N, Suzuki K, Kawachi T, et al. Distribution of marker enzymes and mucin in intestinal metaplasia in human stomach and relation to complete and incomplete types of intestinal metaplasia to minute gastric carcinomas. J Natl Cancer Inst 1980; 65: 231-40.

11 Sipponen P, Kekki M, Siurala M. Atrophic chronic gastritis and intestinal metaplasia in gastric carcinoma. A comparison with a representative population sample. Cancer 1983; 52: 1062-8.

12 Sipponen P. Intestinal metaplasia and gastric carcinoma. Ann Clin Res 1981; 13: 139-43.

13 Murray LA, Williams GT. Sulphomucin-containing intestinal metaplasia in gastric biopsies - one year's experience. [Abstract]. Pathological Society of Gt Britain \& Ireland meeting. January 1984, London. (In press).

14 Jass J. Role of intestinal metaplasia in the histogenesis of gastric carcinoma. J Clin Pathol 1980; 33: 801-10.

15 Wells M, Stewart M, Dixon MF. Mucin histochemistry of gastric intestinal metaplasia. [Abstract]. J Pathol 1982; 138: 70 .

16 Lauren $\mathrm{P}$. The two main histological types of gastric carcinomas; diffuse and so-called intestinal type carcinoma. An attempt at a histoclinical classification. Acta Pathol Microbiol Scand 1965; 64: 31.

17 Ming S-C. Gastric carcinoma. A pathobiological classification. Cancer 1977; 39: 2475-85.

18 Cuello C, Correa P. Dysplastic changes in intestinal metaplasia of gastric mucosa. In: Herpath CL, Sehlag $\mathrm{P}$, eds. Gastric cancer. Heidelberg: Springer-Verlag, 1979: 83-90.

19 Correa P. The epidemiology and pathogenesis of chronic gastritis. Three aetiological entities. In: van der Reis L, ed. Frontiers of gastrointestinal research. Vol 6. Basel: Karger, 1980: 98-108.

20 Kawachi T, Kurisu M, Numanyu N, Sasajima K, Sano $\mathrm{T}$, Sugimura T. Pre-cancerous changes in the stomach. Cancer Res 1976; 36: 2673-7.

21 Cuello C, Correa P, Zamara G, Lopez J, Murray J, Cordillo C. Histopathology of gastric dysplasias. Am J Surg Pathol 1979; 3: 491-500.

22 Crespi M. Premalignant lesions - definitions. In: Cotton PB, ed. Early gastric cancer. Tring: Smith, Kline and French (Proc. of 2nd BSG; SK and F. International Workshop, UK) 1981: 20-4.

23 Jass J, Filipe MI. Sulphomucins and precancerous lesions of the human stomach. Histopathology 1980; 4: 271-9.

24 Jass J. A classification of gastric dysplasia. Histopathology 1983; 7: 181-93.

25 Filipe MI, Bogomoletz V, Dawson P, Fabiani B, Potet $F$. Incidence of intestinal metaplasia subtypes in gastric biopsies collected over a 2-year period: Preliminary data from a joint prospective study in Britain and France. [Abstract]. Path Res Pract 1983; 178: 125.

26 Imai T, Murayama $H$. Time trend in the prevalence of intestinal metaplasia in Japan. Cancer 1983; 2: 353-61. 